\title{
Le narguilé de Gobineau
}

\section{Daniel Bornemann}

\section{(2) OpenEdition}

Journals

Édition électronique

URL : http://journals.openedition.org/rbnu/1214

DOl : $10.4000 /$ rbnu. 1214

ISSN : 2679-6104

\section{Éditeur}

Bibliothèque nationale et universitaire de Strasbourg

\section{Édition imprimée}

Date de publication : 1 novembre 2016

Pagination : 72-75

ISBN : 9782859230630

ISSN : 2109-2761

\section{Référence électronique}

Daniel Bornemann, « Le narguilé de Gobineau », La Revue de la BNU [En ligne], 14 | 2016, mis en ligne le 01 janvier 2020, consulté le 12 décembre 2020. URL : http://journals.openedition.org/rbnu/1214 ; DOl : https://doi.org/10.4000/rbnu. 1214

\section{(C) $10(0$}

La Revue de la BNU est mise à disposition selon les termes de la Licence Creative Commons Attribution - Pas d'Utilisation Commerciale - Partage dans les Mêmes Conditions 4.0 International. 


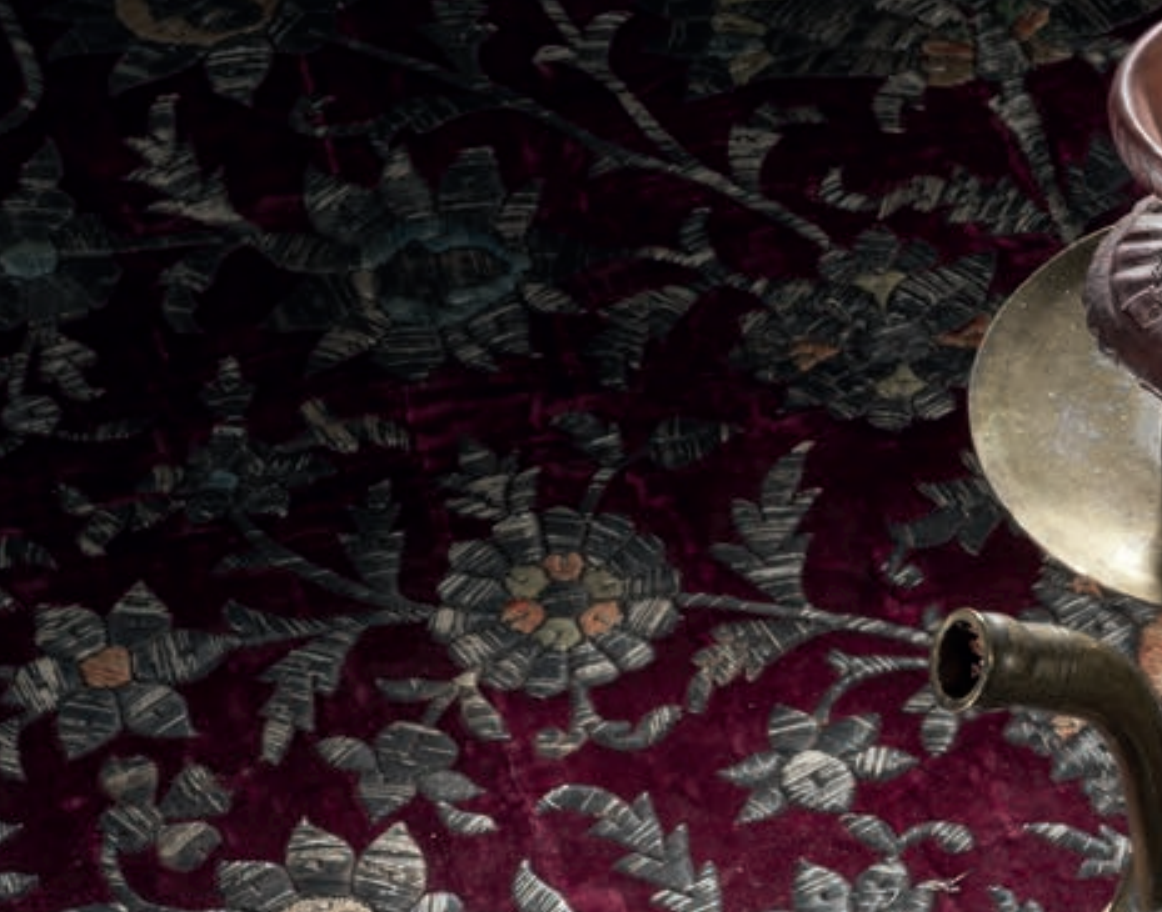
14t.

$-\infty$

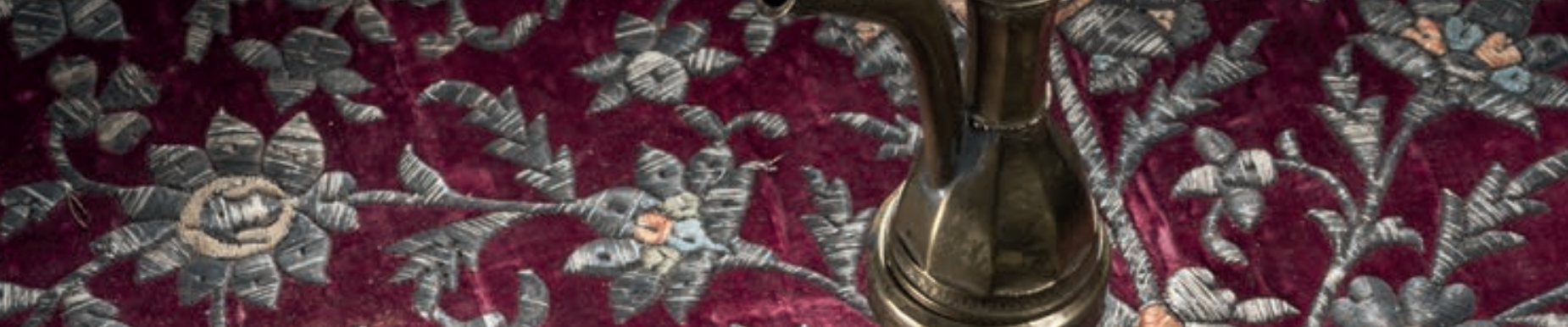



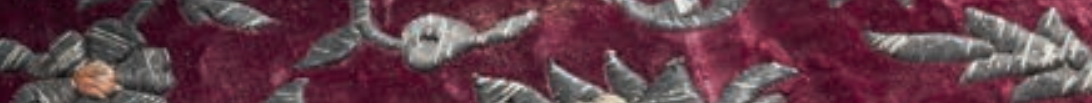





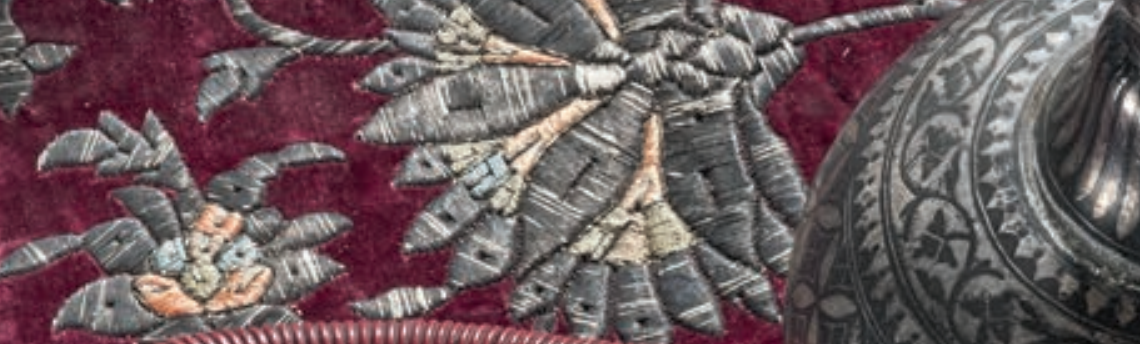

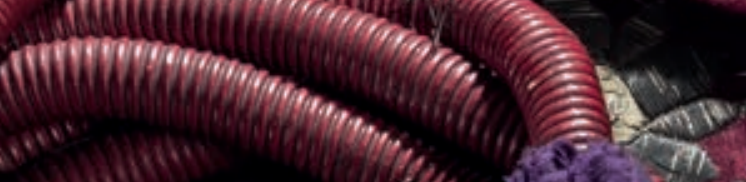

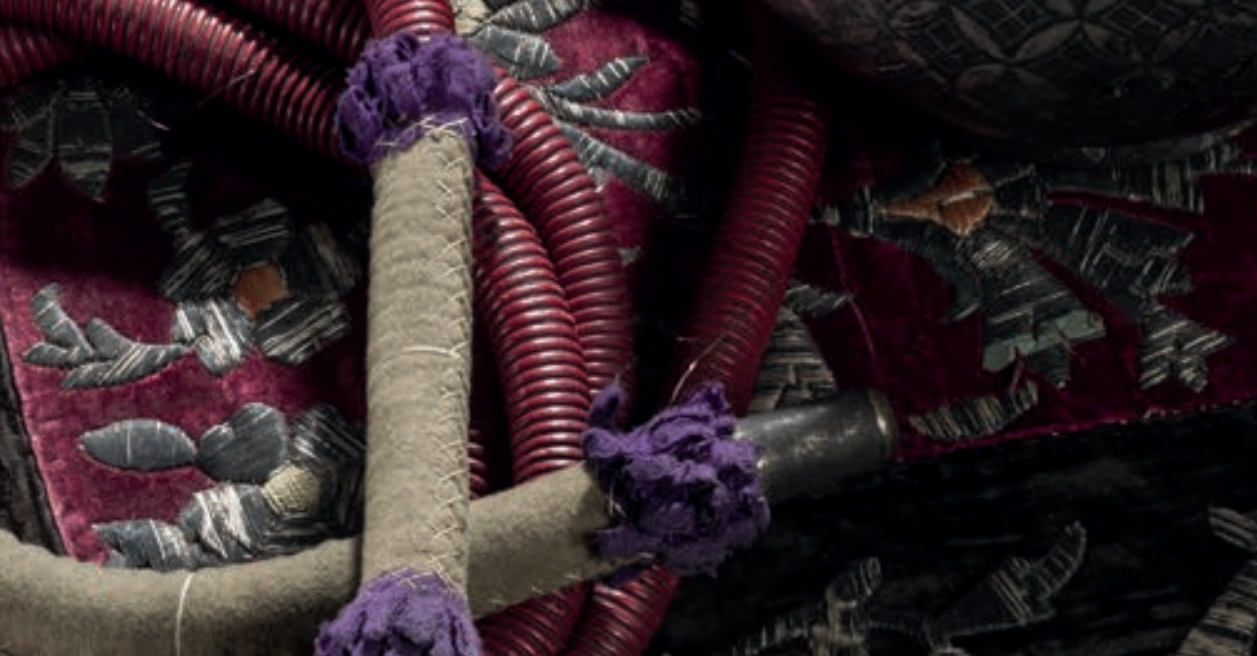

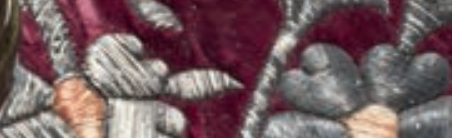

$\frac{4 \pi m 5}{5} \div$

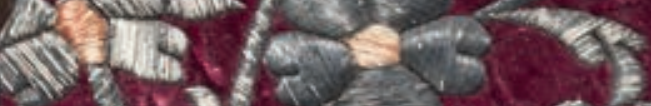
3. 4 है $\%$

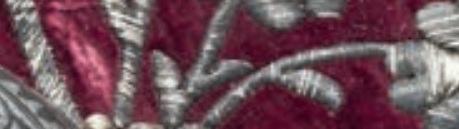

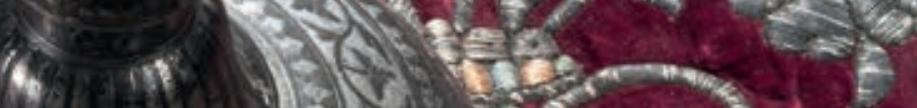
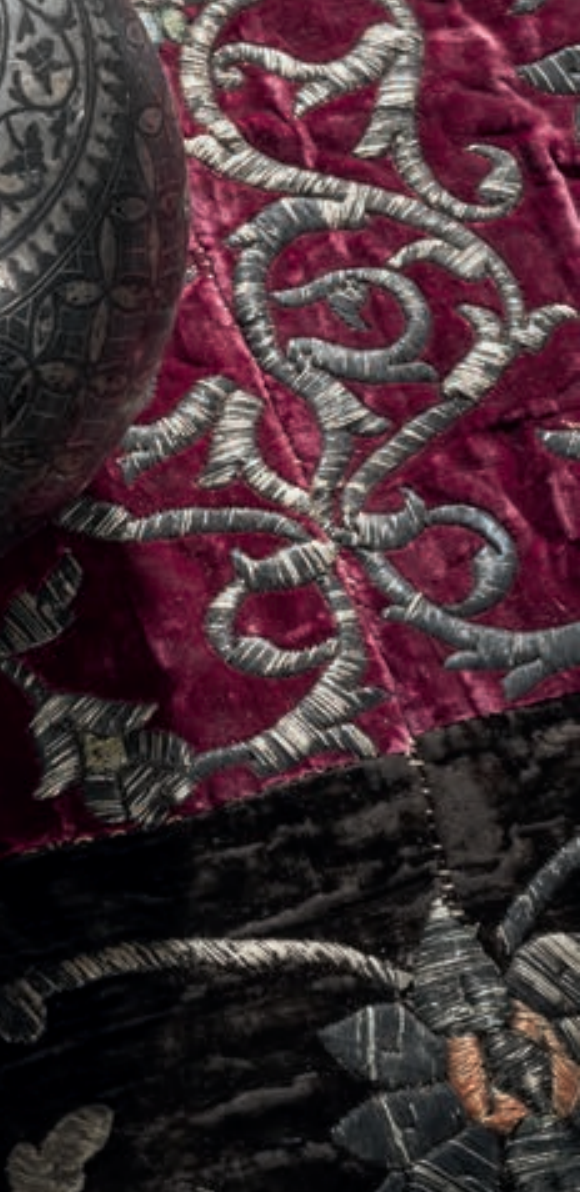
\$) 196 
L'OBJET | Le narguilé de Gobineau

\section{LE NARGUILÉ DE GOBINEAU}

I

1 est des objets que l'on ne s'attend pas à trouver à certains endroits. La présence d'un narguilé dans le fonds de l'écrivain Arthur de Gobineau peut susciter surprise et curiosité. Elle s'explique cependant, et il n'est pas inintéressant de s'attarder un instant sur cet objet. Il n'est pas question ici d'en faire une description scientifique : aux spécialistes de l'ethnographie et de la vie matérielle en Orient au $19^{\mathrm{e}}$ siècle de le faire. Nous proposons plutôt de situer l'objet dans la vie de celui qui le posséda et qui l'utilisa ${ }^{1}$, donc de l'inscrire dans son contexte historique et littéraire.

Ce contexte est celui de la relation de Gobineau à l'Orient, le penseur comptant en effet parmi les orientalistes français du milieu du $19^{\mathrm{e}}$ siècle. L'Orient, en particulier le Moyen-Orient, la Perse, l'Arabie, ont été vus, vécus et étudiés par Gobineau. En témoignent, parmi ses écrits, plusieurs ouvrages : celui qu'il fit paraître en 1858 , sur la Lecture des textes cunéiformes; l'année suivante, en 1859, la parution de son journal de voyage, intitulé Trois ans en Asie, contient toute son aventure orientale. En témoignent également un Traité des écritures cunéiformes paru en 1864, Les religions et les philosophies dans l'Asie centrale, de 1865 , l'Histoire des Perses, de 1869 , et enfin ses Nouvelles asiatiques (1876), un de ses rares succès littéraires.

Ces livres, tout comme son voyage, suivirent de peu la parution de la première partie de l'œuvre qui le rendit, comme on le sait, célèbre : l'Essai sur l'inégalité des races humaines (1853). En effet, en avril 1855, il quitte la France par la mer et atteint l'Arabie par Djeddah, Mascate, Bouchir, pour arriver à Téhéran en juillet. Il y restera trois années, endossant le rôle de premier secrétaire de la légation française. C'est là son premier voyage en Orient. Il en effectue un second entre 1861 et 1863 , où il est envoyé, cette fois-ci en tant que ministre de France, dans les mêmes régions. Le narguilé dont il est question a été acquis lors de ce second voyage. Dans un carnet de notes qui lui est relatif, dans la liste des objets qui remplissent les deux caisses du voyageur, il mentionne un " narghileh " (c'est l'orthographe qu'il utilise) et un " kalian ". L'acquisition du narguilé conservé dans le fonds Gobineau peut être située à Istanbul, comme en atteste une petite étiquette " Narghileh Constantinople " en-dessous du vase. Quant au second, son devenir nous est inconnu.

Nombreux sont les passages de Trois ans en Asie où il est question de l'usage du narguilé : un usage collectif et un rite social. Il fait partie du bon accueil réservé par les Orientaux au secrétaire de légation Gobineau et à son entourage. Il accompagne systématiquement le thé, le café et les sorbets offerts à ces voyageurs de marque. Dans les Nouvelles asiatiques, le rite est également mentionné à plusieurs reprises et évoqué de manière détaillée. "Il n'y a rien de plus agréable au monde que de s'asseoir sous ces abris frais [les feuillages des saules où les oiseaux nichent en foule] en fumant un bon kalian plein de vapeurs odorantes ", dit le héros de la nouvelle intitulée " La guerre des Turcomans ". Gobineau évoque également ce narguilé dans la première de ses Nouvelles asiatiques, "La danseuse de Shamakha ", dont l'histoire est située entre la mer Noire et Bakou.

Houka, chicha, kalian sont autant de dénominations possibles pour cette pipe à eau. Gobineau utilise d'ailleurs 
essentiellement le mot kalian (de l'arabe " ghaliun "). Le mot narguilé (qui peut aussi s'écrire narghilé) vient du persan, et donc, dans le contexte de l'histoire personnelle de Gobineau, c'est peut-être celui qui convient le mieux. L'objet en lui-même est complet, même si certaines parties sont légèrement détériorées. En effet, le foyer en terre cuite (qu'on appelle aussi la douille) est légèrement ébréché. La zone de jointure entre le vase et la colonne a perdu de son étanchéité. Et les tuyaux, qui sont au nombre de quatre, en cuir, sont tous percés par excès d'usure ou de pliures. Le vase a la particularité d'être en métal, probablement en bronze, damasquiné en argent de motifs géométriques. La colonne est en cuivre. Le poids considérable de ce narguilé à vide lui assure certainement une bonne stabilité. Il se rapproche plus du type " égyptien " que du type "syrien ". Sans son foyer, il mesure $39 \mathrm{~cm}$ de haut et son diamètre maximal est de $17,5 \mathrm{~cm}$. Quant au type de décor du vase, il peut être rapproché du style " bidri ", un style décoratif d'origine indienne caractérisé par un fond noir et des motifs plus clairs, serrés, denses, souvent floraux et disposés géométriquement.

À propos des tuyaux, Jean Gaulmier, dans son édition des Nouvelles asiatiques de 1965, affirme qu'en novembre 1873, les sœurs Dragoumis envoient d'Athènes à Gobineau du " tombéky " (tabac) et trois tuyaux pour son narguilé (une bonne réserve), ce qui lui permet de retrouver l'atmosphère persane à Stockholm (où il fut en poste comme ministre plénipotentiaire à partir de 1872). Cela l'a sans doute aidé à écrire ses Nouvelles asiatiques... L'usure des tuyaux, et cette nécessité de les renouveler, semblent en tout cas une preuve de l'usage intense que l'écrivain a fait de cet objet.

Ses théories, qu'elles soient sur les races humaines ou sur les écritures cunéiformes, ne sont plus à prendre au sérieux de nos jours. Sur le second point, elles ne le furent pas même à leur époque de parution. Cependant ses autres écrits orientaux, ses récits de voyages et ses nouvelles gardent à la lecture un charme certain, qui dépasse sans doute celui de ses romans occidentaux ou de ses nouvelles françaises. Arthur de Gobineau est un être aux multiples facettes, et l'image qu'on a de lui pourrait être complétée par celle d'un tranquille fumeur de narguilé ${ }^{2}$.

\section{Daniel Bornemann}

\section{Notes}
1 - Rappelons que la BNU héberge, depuis 1903, le fonds Arthur de Gobineau composé de ses papiers (manuscrits et correspondances), de sa bibliothèque et de meubles et objets divers, dont les sculptures qu'il réalisa à la fin de sa vie. Voir à ce sujet " Le fonds Gobineau ", in Impressions d'Europe : trésors de la BNUS entre France et Allemagne, Strasbourg, BNU, 2003.
2 - Le narguilé de Gobineau est exposé dans les Réserves visitables de la BNU depuis septembre 2015. Son numéro d'inventaire est 2015.1.158. Son inventaire au sein de la collection Gobineau est Varia 34.




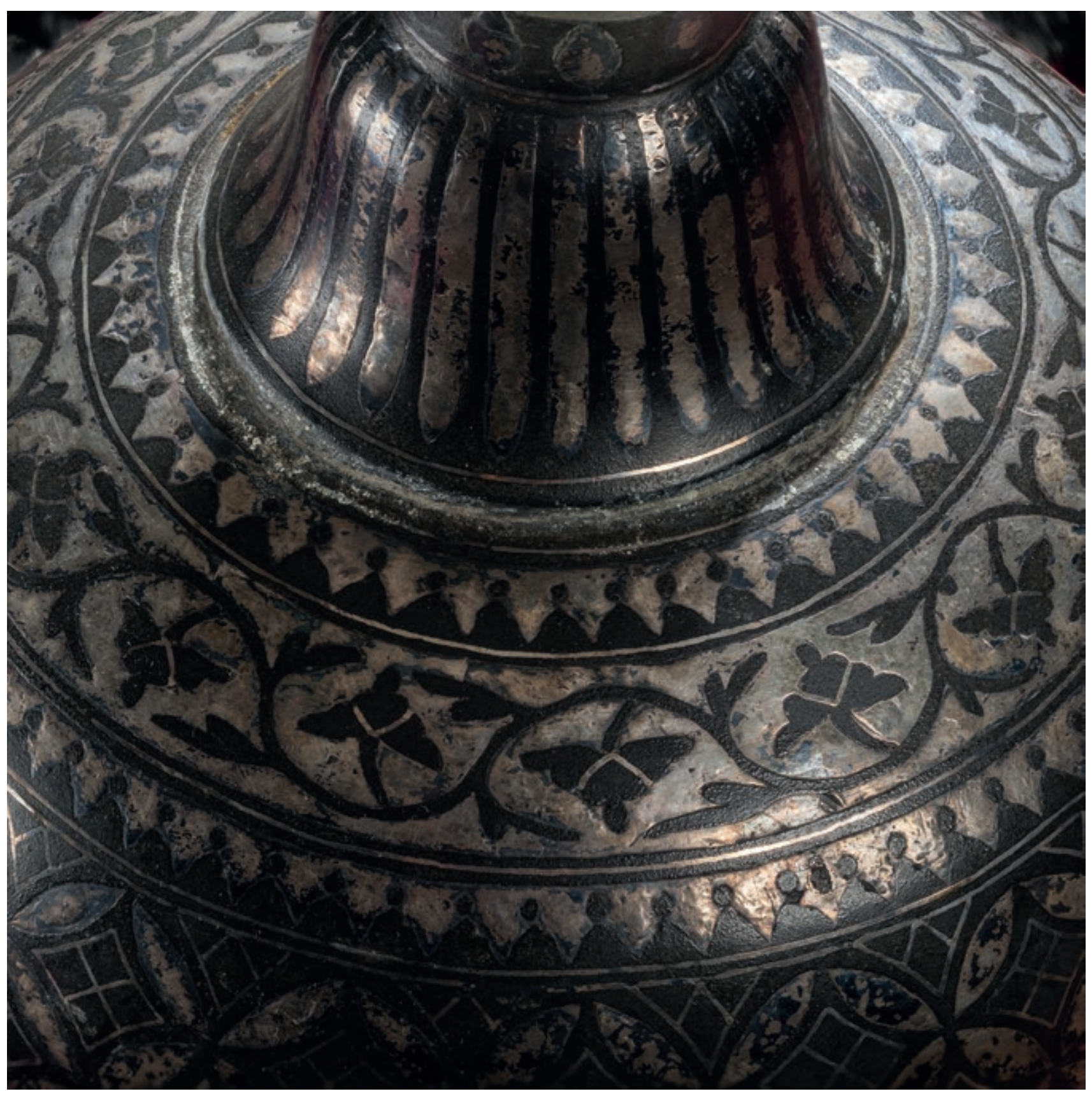

